\title{
Information measures in perceptual experiments: Some pitfalls encountered by Kintz, Parker \& Boynton
}

\author{
A. W. MacRAE ${ }^{1}$ \\ UNIVERSITY COLLEGE OF NORTH WALES
}

The use of information transmission to calculate optimum numbers of response or stimulus categories has the severe disadvantage that empirical information measures are biased estimates of their true values, and the extent of the bias usually depends on the number of stimulus and response categories in use. By neglecting bias, Kintz, Parker, and Boynton have overestimated maximum transmission in their experiments, have wrongly identified the optimal number of response categories, and, by implication, have mistaken the stimulus conditions likely to give greatest transmission.

Kintz, Parker, and Boynton (1969) used information transmission ( $T$ ) calculations to estimate the optimal number of response categories ( $\mathrm{RC}$ ) to be used in identifying spectral hues. They presented a fixed number of hues (21) 10 times each and elicited absolute judgments (AJs) in terms of $8,12,24$, or $44 \mathrm{RCs}$. AJs made with larger numbers of RCs were then collapsed into fewer by grouping RCs, and Ts were calculated for all original and derived sets of judgments. The results showed an asymptotic increase of $\mathrm{T}$ with increasing numbers of RCs.

This result agrees with the conclusions of Eriksen and Hake (1955), who varied the RCs used in judging the sizes of squares and who claimed that increasing the number of RCs increased T. However, it has been shown by MacRae (in press) that the Eriksen and Hake result is an artifact of information bias.

\section{INFORMATION BIAS}

Bias arises primarily because information measures are defined as averages of logarithms of probabilities, but empirical data consist of proportions, which are estimates of these probabilities. The average of the proportions is the best estimate of the average of the probabilities, but the average of the log proportions underestimates the average of the log probabilities. This results in underestimation of information $(\mathrm{H})$ and overestimation of $\mathbf{T}$ (Miller, 1955; Carlton, 1969). In appropriate circumstances, a number of other biases may also occur, and all tend to produce the same result (MacRae, in press). Bias becomes more severe when the number of observations decreases relative to the number of proportions that are used to derive $\mathrm{H}$ or $\mathrm{T}$.

In the case of $T$, this implies an increasing overestimate as the number of stimulus categories (SC) or RC increases, unless the number of observations increases correspondingly. MacRae showed that the frequent experimental finding of an increase of $\mathrm{T}$ with increasing $\mathrm{SC}$ in $\mathrm{AJ}$ experiments resulted from the increase in bias when $\mathrm{SC}$ and $\mathrm{RC}$ increased together, since the total number of judgments either increased proportionately to SC alone or did not increase at all.

A similar effect occurred in the Eriksen and Hake experiment, where SC and RC were varied independently; the result was that conditions with many RCs had inflated $T s$, thus leading to the widely accepted conclusion that it is advantageous to have RC larger than SC. Actually, when bias was allowed for, it was clear that the best $\mathrm{T}$ was given with $\mathrm{SC}$ and $\mathrm{RC}$ equal in number.

\section{KINTZ, PARKER, AND BOYNTON (1969)}

The same kind of bias must operate here since the number of judgments (210) was constant and $\mathrm{RC}$ was increasing. To assess the bias by MacRae's (in press) modification of Carlton's (1969) technique, it is desirable to know the response and error distributions, but it is usually fair to assume a normal distribution of errors and a rectangular distribution of responses. Correction may then be made for the bias in $\mathrm{H}_{(x, y)}$ (which inflates the estimate) and the bias in $\mathrm{H}_{(\mathrm{y})}$ (which diminishes it) since (in the authors' notation) $T_{(x, y)}=H_{(x)}+H_{(y)}-H_{(x, y)}$. The stimulus information, $H_{(x)}$, has no sampling and hence no bias.

The empirical values of $H_{(x, y)}$ and of $\mathrm{H}_{(\mathrm{y})}$ have not been published, but it is possible to calculate their maximum and minimum possible values.

$H_{(x)}$ is constant. $H_{(y)}$ cannot be less than $\mathrm{T}$ and it cannot be greater than $\log _{2} \mathrm{RC}$, where $\mathrm{RC}$ is taken, not as the total number of categories available for response, but as the number shown by their Fig. 2 to have been used by $O$. Taking the smaller value of $H_{(y)}$ underestimates the bias, while the greater one overcorrects.
These corrected Ts are shown in Fig. 1 as stars.

In the condition where 44 categories were used, a more precise calculation is available. Their Fig. 3 shows the uncertainty of $O$ 's responses to each stimulus; the average of these is the response equivocation and is the difference between $\mathrm{H}_{(\mathrm{y})}$ and $\mathrm{T}$. Although only 20 points have been plotted in this figure (the 478 or $486 \mathrm{~nm}$ stimulus having apparently been omitted), it is possible to use it to obtain a much improved bias correction for $T$. These estimates appear in Fig. 1 as circled stars.

Bias estimation has not been attempted for the points that were derived by collapsing together JCs. As the authors say, their after-the-fact groupings probably do not reflect accurately the use that would have been made of these wider categories by $\mathrm{O}$.

\section{DISCUSSION}

Figure 1 shows that the $T$ s obtained in this experiment were subject to considerable bias and that the bias increased as RC increased. Since one $O$ performed just three conditions and the other only two, the optimal size for RC is not certain but it seems likely to agree with the corrected Eriksen and Hake result in being close to $\mathrm{SC}$.

The maximum corrected $T$ is low, corresponding to just over 2 bits, or about five categories correctly identified. SC was

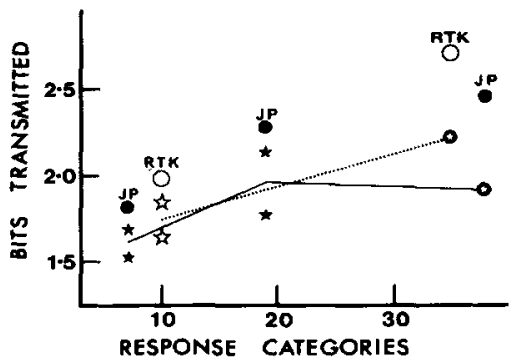

Fig. 1. Original and corrected transmission estimates from an experiment by Kintz, Parker, and Boynton. The circles show the published Ts for two Ss, RTK and JP. The stars show the limits to each corrected $T$, and the circled stars show accurately corrected $T$ s. 
not manipulated as an experimental variable, but MacRae concluded from several such experiments that when $\mathrm{SC}$ and $\mathrm{RC}$ were equal, optimal $\mathrm{T}$ is given with $\mathrm{SC}$ adjusted so that almost all of $H_{(x)}$ is transmitted-that is, by that size of SC where only a few errors are made. This is at variance with the conclusion drawn by Miller (1956) that $T$ increases asymptotically with $\mathrm{SC}$, but this, too, was shown to be an artifact of bias.

Kintz, Parker, and Boynton showed the stimuli in 250-msec flashes and, in these circumstances, errors (or rather, inconsistencies in naming) might be expected to occur with SC quite small.

Thus, not only is it probably incorrect to conclude that the largest $\mathrm{RC}$ gives optimum $\mathrm{T}$ with the 21 wavelengths used here, but it is also incorrect to take even the unbiased maximum as measuring $\mathrm{O}$ 's "channel capacity" for tachistoscopic color flashes in general. Almost certainly, there is some smaller number of stimuli that would permit a larger $T$.

These effects go a long way towards explaining the discrepancies between their result and the earlier work they refer to.

\section{REFERENCES}

CARLTON, A. G. On the bias of information estimates. Psychological Bulletin, 1969, 71, 108-109.

ERIKSEN, C. W., \& HAKE, H. W. Absolute judgments as a function of stimulus range and number of stimulus and response categories. Journal of Experimental Psychology, 1955, $49,323-332$
KINTZ, R. T., PARKER, J. A., \& BOYNTON, R. M. Information transmission in spectral color naming. Perception \& Psychophysics, 1969, 5 241-245.

MacRAE, A. W. Channel capacity in absolute judgment tasks-An artifact of information bias? Psychological Bulletin, in press.

MILLER, G. A. Note on the bias of information estimates. In H. Quastler (Ed.), Information theory in psychology: Problems and methods. Glencoe, Ill.: Free Press, 1955. Pp. 95-100.

MILLER, G. A. The magical number seven, plus or minus two: Some limits on our capacity for processing information. Psychological Review, $1956,63,81-97$.

NOTE

1. Address: University College of North Wales, Bangor, Wales.

(Accepted for publication July 30, 1969.) 